\title{
IDENTIFICACIÓN DE RESTRICCIÓN DEL CRECIMIENTO INTRAUTERINO TARDÍO CON DOPPLER UMBILICAL NORMAL MEDIANTE ALGORITMO DIAGNÓSTICO MULTIPARÁMETRO EN EL INSTITUTO NACIONAL MATERNO PERINATAL
}

\author{
Walter Castillo Urquiaga ${ }^{1}$
}

\begin{abstract}
RESUMEN
Objetivos. Identificar Neonatos con Restricción del Crecimiento Intrauterino (RCIU) Tardío con doppler umbilical normal y evaluar el valor diagnóstico anteparto de un algoritmo multiparámetro. Materiales y métodos. Estudio analítico, observacional y retrospectivo y creación más evaluación preliminar de una prueba diagnóstica. Fueron seleccionados al azar 96 neonatos de 35 a 41 semanas sin anomalías. 23 Pequeños para la Edad Gestacional (PEG) según el método local de clasificación del crecimiento, 13 con peso adecuado para la edad gestacional (AEG) limítrofe (dentro de los $200 \mathrm{~g}$ sobre el percentil 10) y 60 con peso AEG sobre el percentil 25. Se identificaron 22 casos de RCIU (casos) y 74 no RCIU (controles) según una definición operativa pre establecida. Los valores estadísticos fueron calculados para pruebas diagnósticas simples con Epidat 3,1. Resultados. 22 de 96 neonatos(23\% ) fueron clasificados como RCIU; 16 de los 23 PEG (69,5\%); 6 de 13 AEG con peso limítrofe ( 46,2 \%) y 0 de 60 AEG con peso mayor al percentil 25. La sensibilidad, especificidad, VPP, VPN, CP + y CP - global fue de $86,4 \% ; 95,9 \% ; 86,4 \% ; 95,9 \% ; 21,3$ y 0,14 respectivamente. Sólo 4 de 22 (18\%) casos de RCIU fueron diagnosticados como tal en el anteparto a diferencia de 19 de 22 ( 86,4 \% ) mediante el uso del algoritmo diagnóstico. Conclusiones. El uso de un algoritmo diagnóstico multiparámetro mejora la identificación de RCIU en $\geq 35$ semanas con doppler umbilical normal.
\end{abstract}

Palabras clave: Pequeño para la Edad Gestacional; Restricción del Crecimiento Intrauterino; RCIU Tardío; Pequeño Constitucional Normal; Pequeño Sano(fuente: DeCS BIREME).

\section{IDENTIFICATION OF LATE INTRAUTERINE GROWTH RESTRICTION WITH NORMAL UMBILICAL DOPPLER USING MULTIPARAMETER DIAGNOSTIC ALGORITHM IN THE MATERNAL PERINATAL NATIONAL INSTITUTE}

\begin{abstract}
Objectives. To identify late intrauterine growth restricted newborns (IUGR) with normal umbilical artery Doppler and assess the antepartum diagnostic value of a multi parameter algorithm. Material and methods. Analytic, observational, retrospective study and elaboration plus preliminary assessment of a diagnostic tool. 96 non anomalous newborns between 35 and 41 weeks of gestational age were randomly selected. 23 small for gestational age (SGE) according to local growth classification method, 13 borderline adequate for gestational age (AGE)(inside the $200 \mathrm{~g}$ over tenth percentile ) and 60 AEG over the 25 percentil. Using a pre established operative definition 22 IUGR (cases) and 74 non IUGR (controls) were detected. For Statistical analysis we used Epidat 3,1 to calculate values for simple diagnostic Tests. Results. 22 from 96 (23\%) newborns were classified as IUGR; 16 from 23 (69,5\%) SGE , 6 from 13 (46,2\% ) borderline AGE and 0 from AGE over the 25 percentile. Global Sensitivity, Especificity, PPV, NPV, PC + and CP - were 86,4\%; 95,9\%; 86,4\%; 95,9\%; 21,3 and 0,14, respectively. Only 4 from 22 (18\%) cases of IUGR were diagnosed as such in the antepartum unlike 19 from $22(86.4 \%)$ using the diagnostic algorithm. Conclusions. The use of the multi parameter diagnostic algorithm improves the identification of late IUGR fetus with normal umbilical artery Doppler.
\end{abstract}

Key words: Small for gestational age; Intrauterine growth restriction; Late IUGR; Normal constitutionally small; Healthy small (source: MeSH NLM).

\section{INTRODUCCIÓN}

La Restricción del Crecimiento Intrauterino (RCIU) es una importante complicación del embarazo ya que se asocia a un riesgo significativo de morbimortalidad perinatal y consecuencias a largo plazo en la salud ${ }^{1-3}$.

La definición usada para Pequeño para la Edad Gestacional (PEG) se basa en un peso ecográfico estimado o al nacer por debajo del Percentil 10, según una Curva de Crecimiento determinada ${ }^{3-5}$ y hasta la fecha, los términos RCIU y PEG se están usando como sinónimos, prefiriéndose el término último para el periodo post natal; Para el Colegio Americano de Gineco Obstetricia (ACOG) debe reservarse el término RCIU en el anteparto y el de PEG al nacimiento ${ }^{4-6}$. El Royal College de Inglaterra (RCOG) no los diferencia y usa $P E G$ para el feto o neonato pero enfatiza que no es lo mismo Restricción del crecimiento y PEG. Además, el ACOG, a diferencia del RCOG, incluye en su algoritmo diagnóstico los factores de riesgo materno- placentarios y fetales. 
Evidencia actual ${ }^{5-10}$ describe un grupo general que incluye a los Pequeños para la Edad Gestacional y dentro de éstos, se estima que el $50-70 \%$ son Pequeños Constitucionales Normales (PCN), con un crecimiento apropiado para el tamaño materno y la etnicidad con un pronóstico usualmente normal. Otro $10-15 \%$ de los PEG son RCIU (PEG Mediados por la Placenta), o fetos que no han alcanzado su potencial de crecimiento genéticamente determinado debido a una razón patológica o evento en útero que causó una disfunción placentaria en la transferencia de nutrientes. $Y$ por último, otro $5-10 \%$ de los PEG están asociados con anomalías estructurales y cromosómicas, o infección intrauterina (PEG Anormales o No mediados por la Placenta).

En nuestro país los PEG representan un $4 \%$ a $15 \%$ de los embarazos, una frecuencia por debajo de algunos países con semejantes características. García y col ${ }^{11}$ encontró una frecuencia de RCIU de 6,8\% en el Instituto Nacional Materno Perinatal el año 1998 y para el año $2011^{12}$, los PEG representan el $15.9 \%$ de los Egresos Hospitalarios en neonatología en este hospital y no hay datos exactos de la frecuencia de verdaderos RCIU.

Evidencia actual demuestra que hay dos grupos diferentes de $\mathrm{RCIU}$, los de aparición temprana ( $\leq 34$ semanas) con alteración común en el doppler umbilical ${ }^{1-9}$ y los de aparición tardía ( $\geq 35$ semanas) que tienen una diferente presentación ${ }^{14-17,}$ 18-21.

Así, la discriminación entre los grupos de fetos afectados en su crecimiento considerando su diferente presentación, es esencial desde una óptica clínica ya que van a presentar diferencias en el pronóstico, evolución y manejo antenatal y post natal y por tanto la identificación de los fetos con RCIU de presentación tardía es retadora y no puede basarse sólo en el doppler de la arteria umbilical $1,3,5,9,10,14,20$.

En el presente estudio exploramos la hipótesis que el uso de múltiples parámetros a través de un algoritmo prediseñado podría identificar antenatalmente fetos con RCIU con doppler umbilical normal en riesgo de pronóstico perinatal adverso.

\section{MATERIALES Y MÉTODOS}

Se realizó un estudio Analítico, Observacional y Retrospectivo y de Creación y evaluación Preliminar de un Instrumento diagnóstico. Se usó el tamaño de muestra para estudios descriptivos con prevalencia de 6,8 \% y para propósito de aplicación de prueba diagnóstica se acortó al $30 \%$. Se seleccionaron al azar 23 neonatos Pequeños para la Edad Gestacional según el método tradicional de clasificación del crecimiento, 13 con peso adecuado para la edad gestacional pero limítrofe ( dentro de los $200 \mathrm{~g}$ sobre el percentil 10) y 60 con peso adecuado para la edad pero sobre el percentil 25 según dicho método. Se identificaron 22 casos de RCIU (casos) y 74 no RCIU (controles) según una definición operativa pre establecida para propósito del estudio.
Los criterios de inclusión fueron Recién Nacidos Vivos de Gestación única con Edad gestacional entre las 35 - 40 semanas según la datación realizada por el neonatólogo e historia clínica materna y neonatal accesible e información requerida completa. Además, tener una evaluación ecográfica doppler umbilical normal dentro de la semana al parto. Fueron excluidos aquellos con Edad gestacional menor de 35 semanas, con malformación congénita o cromosomopatía conocida o sospechada, embarazo múltiple, presencia de infección por Toxoplasma o Citomegalovirus, Virus de la Inmunodeficiencia Humana y Tuberculosis, Ruptura Prematura de Membranas e historia clínica no disponible y/o con información requerida incompleta.

En la población seleccionada se revisó la historia clínica materna y las evaluaciones ecográficas con la finalidad de determinar la edad gestacional más exacta al nacimiento, se aplicó la Curva de Crecimiento Intrauterino de Ticona y sus factores de corrección para la clasificación de la adecuación del crecimiento en relación al peso neonatal y se elaboró un Instrumento con factores e indicadores con definiciones operacionales pre establecidos para el diagnóstico operativo de RCIU el cual implicó cualquiera de lo siguiente:

a. Peso < Percentil 2,5 con/sin presencia de factores maternos que afecten la Transferencia de nutrientes o indicadores de transtorno de la placentación, de compromiso hemodinámico o de bienestar fetal anormal.

b. Peso < Percentil $10+$ (factor Materno que afecte la transferencia de nutrientes ó indicador de transtorno de la placentación o indicador de compromiso hemodinámico fetal o indicador de bienestar fetal anormal).

c. Peso $>\mathrm{P} 10+$ indicador de compromiso hemodinámico fetal

Posteriormente, se aplicó el algoritmo diagnóstico al día de la última evaluación ecográfica determinando como positividad de la prueba al diagnóstico de RCIU mediante dicho algoritmo. Los valores estadísticos para evaluación de la prueba diagnóstica fueron calculados con Epidat 3,1.

\section{RESULTADOS}

Tabla 1. Evaluación del algoritmo diagnóstico anteparto en 23 neonatos pequeños para la edad gestacional por Capurro-INPROMI

\begin{tabular}{|c|c|c|c|c|}
\hline \multicolumn{5}{|c|}{$\begin{array}{l}\text { Nivel de confianza: } 95,0 \% \\
\text { Diagnóstico Post natal }\end{array}$} \\
\hline Algoritmo anteparto & RCIU & NO RCIU & Total & S: $87,5 \%$ \\
\hline---- & ----- & - ------ & & E: $85,7 \%$ \\
\hline Positivo & 14 & 1 & 15 & VPP: $93,3 \%$ \\
\hline Negativo & 2 & 6 & 8 & VPN : $75 \%$ \\
\hline & $\cdots$ & -- & $\cdots$ & Prev : $69 \%$ \\
\hline \multirow[t]{2}{*}{ Total } & 16 & 7 & 23 & $\mathrm{CP}+: 6,13$ \\
\hline & & & & $\mathrm{CP}-: 0,15$ \\
\hline
\end{tabular}


Tabla 2. Evaluación del Algoritmo Diagnóstico anteparto en 13 neonatos con peso para la edad gestacional limítrofe (AEG + $200 \mathrm{~g}$ ) por Capurro-INPROMI

\begin{tabular}{|c|c|c|c|c|}
\hline \multicolumn{5}{|c|}{$\begin{array}{l}\text { Nivel de confianza: } 95,0 \% \\
\text { Diagnóstico Posnatal }\end{array}$} \\
\hline Prueba diagnóstica & RCIU & NO RCIU & Total & S : $83,3 \%$ \\
\hline Positivo & 5 & 1 & 6 & VPP: $83 \%$ \\
\hline Negativo & 1 & 6 & 7 & VPN: $85,7 \%$ \\
\hline Total & 6 & 7 & 13 & $\begin{array}{l}C P+: 5,8 \\
C P-: 0,19\end{array}$ \\
\hline
\end{tabular}

Tabla 3. Evaluación del Algoritmo Diagnóstico anteparto en 60 neonatos con peso adecuado para la edad gestacional (>Percentil 25) por Capurro -INPROMI

\begin{tabular}{|c|c|c|c|c|}
\hline \multicolumn{5}{|c|}{$\begin{array}{l}\text { Nivel de confianza: } 95,0 \% \\
\text { Diagnóstico Post natal }\end{array}$} \\
\hline Algoritmo anteparto & RCIU & NO RCIU & Total & S: $50 \%$ \\
\hline Positivo & 0 & 1 & 1 & VPP: 33,3 \\
\hline Negativo & 0 & 59 & 59 & VPN: $98,4 \%$ \\
\hline \multirow{2}{*}{ Total } & 0 & 60 & 60 & \\
\hline & 0 & 60 & 60 & $\mathrm{CP}-: 0,5$ \\
\hline
\end{tabular}

Tabla 4. Evaluación del Algoritmo Diagnóstico anteparto en 96 neonatos totales seleccionados

\begin{tabular}{|c|c|c|c|c|}
\hline \multicolumn{5}{|c|}{ Nivel de confianza: $95,0 \%$} \\
\hline Algoritmo anteparto & $\mathrm{RCIU}$ & NO RCIU & Total & E: $95,9 \%$ \\
\hline Positivo & 19 & 3 & 22 & VPN: $95,9 \%$ \\
\hline Negativo & 3 & 71 & 74 & Prev : 22,9 \\
\hline Total & 22 & 74 & 96 & $\mathrm{CP}_{-}: 0,14$ \\
\hline
\end{tabular}

\section{DISCUSIÓN}

Nuestros hallazgos confirman la hipótesis que el uso de múltiples parámetros en un Algoritmo optimizan la detección de RCIU tardío con doppler umbilical normal. Las significativas Sensibilidad, Especificidad y Coeficientes de Probabilidad muestran la utilidad de dicha prueba diagnóstica en el anteparto. Adicionalmente, sólo 4 de 22 ( 18\%) casos de RCIU fueron diagnosticados como tal en el anteparto a diferencia de 19 de 22 ( 86,4 \% ) mediante el uso del Algoritmo diagnóstico.

Se postula que el deficiente diagnóstico y diferenciación entre PEG, RCIU y PCN como demuestra este estudio se debe en parte a la diferente Curva de Crecimiento Intrauterino ${ }^{22-26}$ y a las Imprecisas determinaciones de la Edad Gestacional ${ }^{3,4,9,10}$. Las Curva de Crecimiento de Ticona ${ }^{27}$ mejora la detección de RCIU optimizando la distinción entre la pequeñez fisiológica y la patológica (RCIU) $)^{1,3,13,20}$. La valoración de edad por examen físico es de ayuda cuando no hay evidencia para una datación correcta, pero puede subdiagnosticar RCIU. La curva de crecimiento de INPROMI usada en nuestra institución al parecer está subvalorando la verdadera prevalencia de RCIU.

Hemos hallado numerosos fetos no identificados como PEG y más aún fetos con doppler umbilical normal están siendo considerados fetos Pequeños Constitucionales Normales o Pequeños Sanos sin considerar otros parámetros o la diferente presentación que depende de la edad gestacional y de la severidad del compromiso. Está descrito que los RCIU de presentación tardía (> 34 semanas ) que son más frecuentes, generalmente no presentan compromiso hemodinámico ni asociación a pre eclampsia y por tanto son subdiagnosticados. La evaluación doppler de la arteria cerebral media, el Índice Cerebro Placentario y de la arterias uterinas, la existencia de un factor materno o indicador de afección de la transferencia de nutrientes mediados por la placenta o un déficit severo del crecimiento (menor del percentil 2,5-3) mejoran su identificación y por tanto, la normalidad del doppler umbilical no debe incitar a definirlos como simples variantes extremos de lo normal ya que estos también tienen peores pronósticos perinatales y neuroconductuales ${ }^{18-20,28-32}$.

La importancia de este estudio radica en poder establecer criterios o algoritmos que permitan una identificación anteparto y postparto más certera de fetos pequeños para la edad gestacional y en especial fetos con verdadera restricción del crecimiento intrauterino en mayor riesgo de morbimortalidad y de secuelas a largo plazo.

Detectar los PEG y diferenciar los RCIU de los pequeños constitucionales permitirá preveer manejos y evitar desenlaces a corto plazo como el evitar las muertes intra útero, a mediano plazo en relación a las complicaciones neonatales inmediatas y mediatas que causan internamiento y costos hospitalarios y aun muerte neonatal y a largo plazo porque una intervención temprana podría evitar secuelas como aquellas sobre el neurodesarrollo.

Aunque la condición de alto riesgo de nuestras pacientes y ser centro de referencia haya motivado la alta prevalencia de RCIU en los casos encontrados; no desvalora la utilidad de la prueba diagnóstica ya que además muestra una alta especificidad en neonatos y fetos normales. Probablemente un estudio prospectivo y multicéntrico permita una validación y una aplicabilidad que permita valorar el impacto en el pronóstico perinatal.

El doppler umbilical no es confiable para la identificación de RCIU tardío el cual es más prevalente y de más dificil 
diagnóstico y el uso de un algoritmo multiparámetro mejoró la detección y se propone como una herramienta diagnóstica prometedora para la identificación anteparto.

\section{REFERENCIAS BIBLIOGRAFICAS}

1. Figueras $F$ and Gardosi J. Intrauterine growth restriction: new concepts in antenatal surveillance, diagnosis, and management. Am J Obstet Gynecol 2011; 204 (4): 288 - 300

2. Chernausek S. Update: Consequences of abnormal fetal growth. J Clin Endocrinol Metab 2012; 97:689-695.

3. Mayer $\mathrm{C}$ and Joseph K. Fetal growth : a review of terms, concepts and issues relevant to obstetrics. "Accepted article", doi:10.1002/uog.11204 Ultrasound Obstet Gynecol 2012

4. Chauhan SP, Gupta LM, Hendrix NW, et al. Intrauterine growth restriction: comparison of American College of Obstetricians and Gynecologists Practice Bulletin with other national guidelines. Am J Obstet Gynecol 2009;200:409.e1409.e6.

5. Royal College of Obstetricians and Gynecologists The investigation and management of the small-for-gestatationalage fetus. Guideline No. 31. London, UK: Royal College of Obstetricians and Gynecologists; 2002.

6. American College of Obstetricians and Gynecologists. Intrauterine growth restriction. Washington, DC: American College of Obstetricians and Gynecologists; 2000

7. Intrauterine Growth Restriction. Clinical guideline Date Revised: December 2010 King Edward Memorial Hospital Review Team: OGCCU Perth Western Australia

8. Small for gestational age. Clinical guideline Date Revised: December 2010 King Edward Memorial Hospital Review Team: OGCCU Perth Western Australia

9. Sanín-Blair JE y col. Diagnosing and managing foetuses suffering from intrauterine growth restriction (IUGR) and foetuses which are small for their gestational age (SGA). Colombian consensus. Revista Colombiana de Obstetricia y Ginecología 2009; 60 (3): 247-261

10. Fescina RH y col. Vigilancia del Crecimiento fetal 2da ed. Publicación Científica OPS CLAP/SMR 15862011

11. García P, Ruiz C, Huaman I. Incidencia y Factores asociados con el retardo de Crecimiento Intrauterino. Estudio de casos y controles. XIII Congreso Peruano de Obstetricia y Ginecología, 2000

12. Instituto Nacional Materno Perinatal. Reporte estadístico. Consolidado anual 2011 (on-line). (PERU): <iemp.gob.pe>

13. Kase BA, Carreno CA, Blackwell SC. Customized estimated fetal weight: a novel antenatal tool to diagnose abnormal fetal growth. Am J Obstet Gynecol 2012;207

14. Crispi $F$ y col. Placental angiogenic growth factors and uterine artery Doppler findings for characterization of different subsets in preeclampsia and in isolated intrauterine growth restriction. Am J Obstet Gynecol 2006; 195 (1): 201-7

15. Cruz M, Gao W and Hibbard J. Obstetrical and perinatal outcomes among women with gestational hypertension, mild preeclampsia, and mild chronic hypertension. Am J Obstet Gynecol 2011; 205: 260e1-9.

16. Savchev $S$ and col. Estimated weight centile as a predictor of perinatal outcome in small-for-gestational-age pregnancies with normal fetal and maternal Doppler indices. Ultrasound Obstet Gynecol 2012; 39: 299-303.

17. Brosens I, Pijnenborg R, Vercruysse L and Romero R. The "Great Obstetrical Syndromes" are associated with disorders of deep placentation. AmJ Obstet Gynecol 2011;204(3):193201

18. Cruz-Martinez R y col. Cerebral blood perfusión and neurobehavioral performance in full term small for gestational age fetuses. Am J Obstet Gynecol 2009; 201(5): 474 e1-7

19. Cruz-Martinez $R$ y col. Fetal brain Doppler to predict cesarean delivery for non-reassuring fetal status in term, small-for gestational age fetuses. Obstet Gynecol 2011;117: 618-26.

20. Baschat A. Neurodevelopment following fetal growth restriction and its relationship with antepartum parameters of placental dysfunction. Ultrasound Obstet Gynecol 2011; 37 : 501-514

21. Odibo A, Cahil A, Odibo L, Roehl K and macones G. Prediction of intrauterine fetal death in small-forgestational- age fetuses: impact of including ultrasound biometry in customized modelsUltrasound Obstet Gynecol 2012; 39: 288-292

22. Hernández J, Acosta M, Maldonado C, Sacieta L, Meza A. Curva de crecimiento intrauterino. Pediatría UNMSM. 1976;1(1):7-18.

23. González R y col. Curva nacional de distribución de peso al nacer según edad gestacional. Chile, 1993 a 2000. Rev Méd Chile 2004; 132: 1155-1165.

24. Ticona M, Huanco D. Crecimiento intrauterino en un grupo seleccionado de recién nacidos de Tacna. Rev Peru Ginecol Obstetr 2002; 48 (2): 93-9.

25. Velásquez P. Evaluación de las curvas de crecimiento intrauterina usadas en el Perú. [Tesis de Especialista en Neonatología]. Facultad de Medicina Humana, Universidad Nacional Mayor de San Marcos; 2003

26. Parra L, Hermosa S, Dávila R, Parra J, Chumbe O, Orderique. Curvas de crecimiento intrauterino en una población de recién nacidos peruanos. Resúmenes del XXIII Congreso Peruano de pediatría. Trujillo, 2004

27. Ticona M, Huanco D. Curvas de crecimiento intrauterino propias del Perú y su efecto en la identificación de una nueva población neonatal de riesgo nutricional. Serie Informes Técnicos $N^{\circ} 73$, MINSA INS 2007

28. Simic M, Amer-Wahlin I, Marsal K and Kallén. Effect of various dating formulae on sonographic estimation of gestational age in extremely preterm infants. Ultrasound Obstet Gynecol 2012; 40: 179-185

29. Sanz - Cortés M and col. Abnormal brain microstructure and metabolism in small-for-gestational-age term fetuses with normal umbilical artery Doppler. Ultrasound Obstet Gynecol 2010; 36: 159-165.

30. Chander L and Sonal G. Colour Doppler in IUGR- where are we and where do we go?. J Obstet Gynecol India 2010, 60(4): 301-311.

31. Gómez $O$ and col. Sequential changes in uterine artery blood flow pattern between the first and second trimesters of gestation in relation to pregnancy outcome. Ultrasound Obstet Gynecol 2006; 28:802-808.

32. Souka $A$ and col. Performance of third-trimester ultrasound for prediction of small-for-gestational-age neonates and evaluation of contingency screening policies. Ultrasound Obstet Gynecol 2012; 39:535-542. 